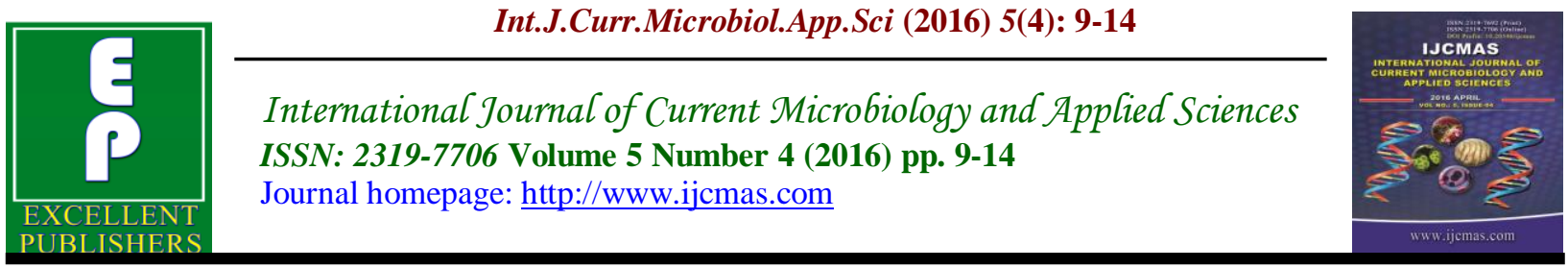

Original Research Article

http://dx.doi.org/10.20546/ijcmas.2016.504.002

\title{
Effect of Low Energy Surface Vibration on the Anti-adhesive Properties of Multipurpose Solutions
}

\author{
Shilpa Sabnis* and Tejas Nair \\ Department of Microbiology, The Institute of Science, Mumbai, India \\ *Corresponding author
}

\begin{abstract}
A B S T R A C T
Keywords

Contact lenses,

Ocular isolates,

MPS, Low energy

surface vibrations,

Biofilm,

Anti-adhesive,

Antimicrobial.

Article Info

Accepted:

08 March 2016

Available Online:

10 April 2016

Proper cleaning and disinfection of contact lenses is critical to ensuring successful lens wear by eradicating debris and potentially pathogenic microorganisms from the lens surface. In addition, these contemporary Multipurpose Solutions (MPS) have also simplified lens care for patients by incorporating various components that are gentle to ocular tissues and are capable of performing the essential functions of cleaning and disinfecting within a single formulation with protein removal being an added attribute at times in many cases. Low-energy surface acoustic waves can inactivate bacteria and deagglomerate bacterial clusters or flocks. The present study was therefore, an attempt to determine the effect of low energy surface vibrations on biofilm formation by four different ocular isolates viz. Pseudomonas aeruginosa, Candida albicans, Klebsiella pneumoniae, and Staphylococcus aureus in the presence of three commercially used Multipurpose Solutions namely, Biotrue, Aquasoft and ReNu. The use of low energy surface vibrations along with MPS was found to aid the MPS in their anti-adhesive as well as antimicrobial actions.
\end{abstract}

\section{Introduction}

Contact lenses are a successful form of vision correction. But their use has been associated with an increased risk of microbial keratitis (MK), contact lens related acute red eye (CLARE) as well as contact lens peripheral ulcer (CLPU) (Eltis, 2011). One of the main problems associated with contact lenses is microbial contamination of the lens surface. Microbial contamination further leads to the formation of biofilm by potentially pathogenic microorganisms on the surface of the contact lens. Proper cleaning and disinfection of contact lenses is critical to ensuring successful lens wearing. One of the main problems associated with contact lenses is microbial contamination of the lens surface. It is necessary to eradicate debris and potentially pathogenic microorganisms from the lens surface. Despite the simplification of lens care regime, no protection from infection other than regular cleaning and disinfection is provided with normal cleaning solutions.

The International Organization for Standardization (ISO) has established microbiological requirements and test methods for products and regimens for hygienic management of contact lenses with 
methodology and acceptance criteria for standalone disinfecting solutions (ISO/CD14729). According to this standard, the disinfecting solution must be able to reduce Staphylococcus aureus, Pseudomonas aeruginosa by 3 log units and Candida albicans by $1 \log$ unit at the minimum disinfection time recommended by manufacturers.

Low-energy surface acoustic waves generated from electrically activated piezo elements are shown to effectively prevent microbial biofilm formation on indwelling medical devices. In medicine, ultrasound transducers are used for diagnosis of abnormalities and fetal, abdominal, and heart diseases. Ultrasound has been applied in microbiology for sonication and inactivation of bacteria in food processing. However, the effect of ultrasound has mainly been studied for food- and waterassociated bacteria, and data are lacking for clinically important species, such as Staphylococci, enterococci, Haemophilus influenzae, and Pseudomonas aeruginosa (Duckhouse et al., 2004; Kordowska-Wiater and Stasiak, 2011).

The present study was therefore aimed at determination of the effect of low energy surface vibrations on biofilm formation by four different ocular isolates viz. Pseudomonas aeruginosa, Candida albicans, Klebsiella pneumoniae, and Staphylococcus aureus in the presence of three commercially used Multipurpose Solutions namely, Biotrue, Aquasoft and ReNu.

\section{Materials and Methods}

Effect of Low Energy Surface Vibration Wave on Anti-adhesive Property of Multipurpose Solutions (MPS)

Eighteen hour old suspensions of
Pseudomonas aeruginosa, Candida albicans, Klebsiella pneumoniae, and Staphylococcus aureus (cell density $10^{8}$ cells/ml) were taken in two sets. Set A was treated with low energy surface vibrations wave whereas set B was used as control. Contact lenses as an adhesive support were placed in glass tubes containing three different commercially available Multipurpose Solutions (MPS) viz. Biotrue, Aquasoft and $\mathrm{ReNu}$ and incubated under static conditions at $36 \pm 1^{\circ} \mathrm{C}$ for $6 \mathrm{~h}$. Each lens was washed three times with sterile distilled water to remove non-adherent bacteria, placed in a glass tube containing Phosphate Buffered Saline ( $\mathrm{pH}$ 7.2). Tubes from Set A were sonicated briefly at 40 $\mathrm{kHz} / 3 \mathrm{~min}$ in an ultrasonic cleanser whereas Set B was maintained under static conditions. Smears taken from the individual lens surface were then added to $1 \mathrm{ml}$ Phosphate Buffered Saline ( $\mathrm{pH}$ 7.2). The solutions from each tube were then individually plated on sterile nutrient media viz. St. Nutrient Agar (Pseudomonas aeruginosa, Klebsiella pneumoniae, and Staphylococcus aureus) and st. Saboraud's Agar (Candida albicans) and the results were expressed as $\mathrm{cfu} / \mathrm{ml}$ of adhered organisms.

\section{Results and Discussion}

\section{Effect of Low Energy Surface Vibration Wave on Anti-adhesive Property of MPS Solutions}

In this study, we tried to examine the effects of low energy surface vibration wave on biofilm formation by four different ocular isolates (obtained from the surfaces of various contact lenses) in presence of all the three different commercially available test MPS (viz. Bio True, Aqua soft and ReNU). All the three MPS were found to bring about a reduction in the number of organisms on the surface of contact lenses. $\mathrm{ReNu}$ was 
found to be the most effective of the three, in fact bringing about a complete removal of Candida albicans from the lens surface on treatment with MPS accompanied with low energy surface vibrations. Of all the isolates used in the study, the order of sensitivity was found to be Candida albicans > Pseudomonas aeuginosa $>$ Staphylococcus aureus $>$ Klebsiella pneumoniae. The results obtained are in accordance with earlier reports where Gram positive organisms are comparatively more resistant to low energy surface vibrations in comparison with Gram negative organisms (Monsen et al., 2009). Gram-negative microorganisms, having an outer membrane, are usually susceptible to limited sonication. However, in spite of being a Gram negative organism, Klebsiella pneumonia was found to be the most resistant of the four ocular isolates used in the study. Presumably, the outer capsular polysaccharide of Klebsiella pneumoniae must have resulted in its increased resistance to sonication.

Table.1 Effect of Low Energy Surface Vibrations Wave on Anti-adhesive Property of MPS Solutions in Terms of cfu/ml ofAadhered Organisms

\begin{tabular}{|c||c|c|c|c|c|c|}
\hline \multirow{2}{*}{ MultiPurpose solutions } & \multicolumn{4}{|c|}{ COLONY FORMING UNIT (CFU/ml ) } \\
\cline { 2 - 7 } & \multicolumn{2}{|c|}{ BioTrue } & \multicolumn{2}{c|}{ Aqua soft } & \multicolumn{2}{c|}{ ReNu } \\
\cline { 2 - 7 } & A & B & A & B & A & B \\
\hline \hline Pseudomonas aeruginosa & 19 & 10 & 25 & 9 & 11 & 5 \\
\hline Candida albicans & 9 & 2 & 12 & 5 & 3 & 0 \\
\hline Staphylococcus aureus & 30 & 12 & 23 & 10 & 42 & 35 \\
\hline Klebsiella pneumoniae & 34 & 2 & 50 & 26 & 22 & 9 \\
\hline
\end{tabular}

Key: A= only MPS

$B=$ MPS with low-energy surface vibration wave

Fig.1 Effect of Low Energy Surface Vibration Wave on Anti-Adhesive Property of MPS on Pseudomonas Aeruginosa Biofilms

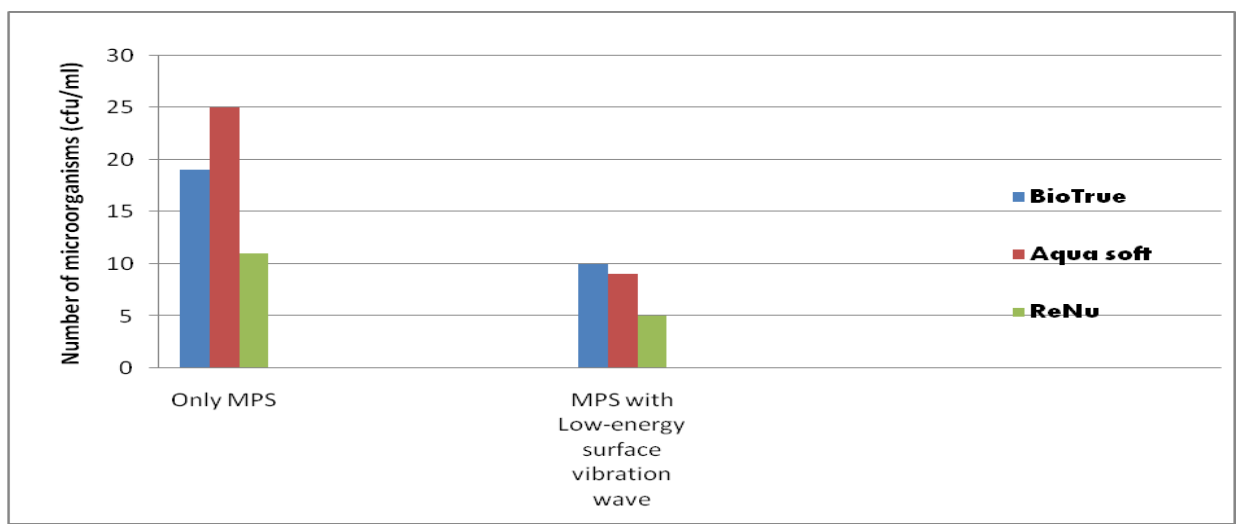


Fig.2 Effect of Low Energy Surface Vibration Wave on Anti-Adhesive Property of MPS on Candida albicans Biofilms

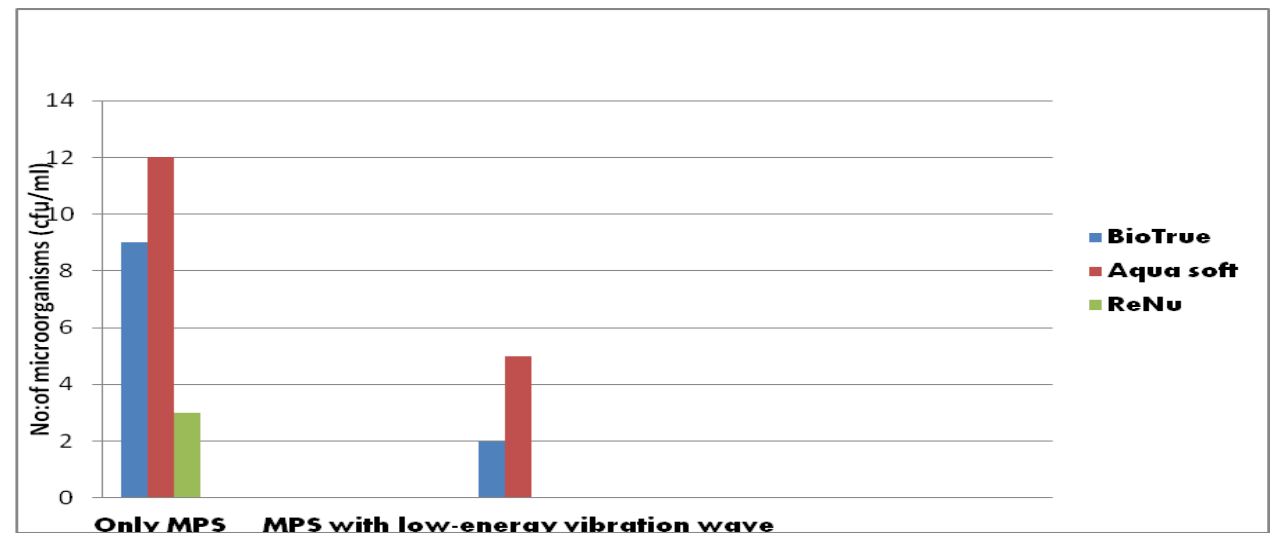

Fig.3 Effect of Low Energy Surface Vibration Wave on Anti-Adhesive Property of MPS on Klebsiella pneumoniae Biofilms

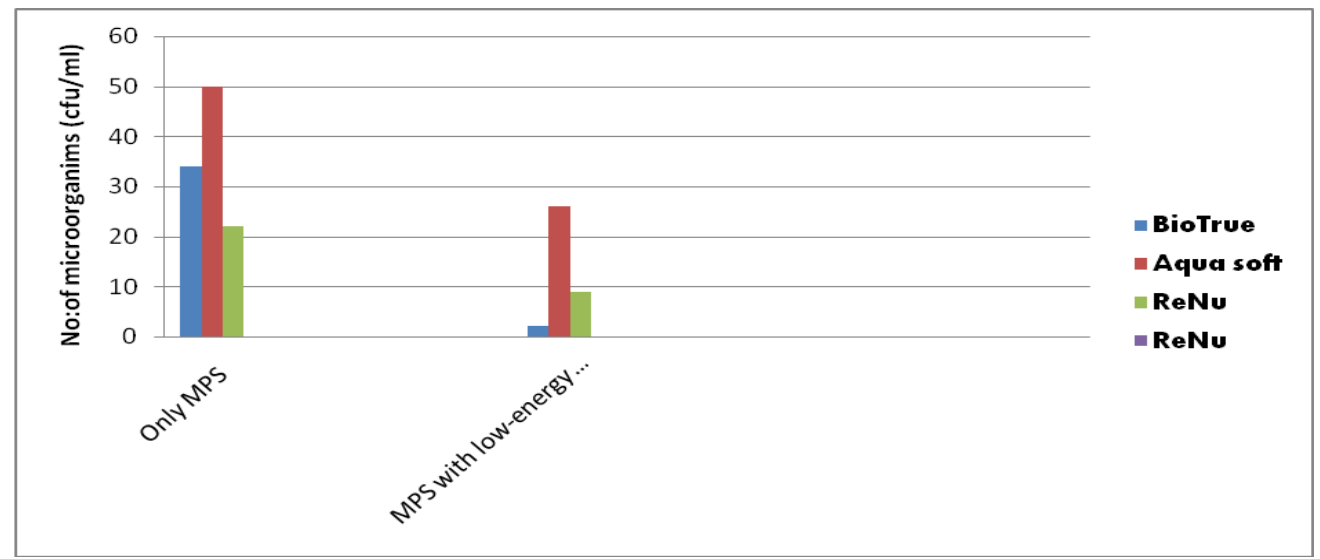

Fig.4 Effect of Low Energy Surface Vibration Wave on Anti-Adhesive Property of MPS on Staphylococcus aureus Biofilms

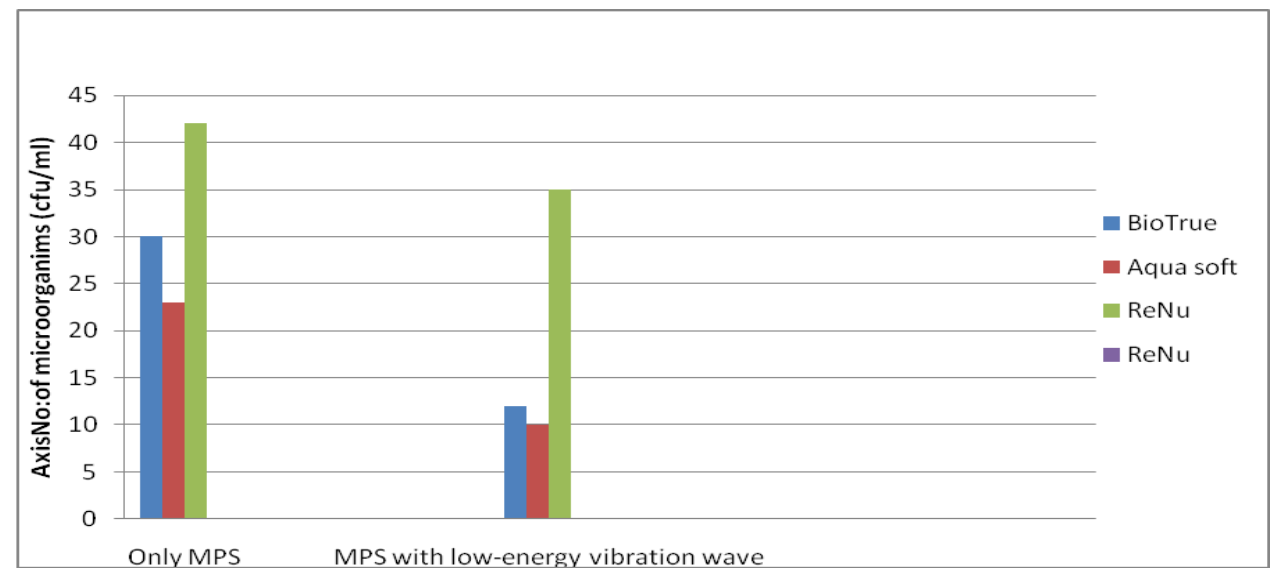


It has been reported in a study that the biofilm development by all the bacteria as well as Candida species is prevented when such elastic waves with amplitudes in the nanometer range are applied (Hazan et al., 2006). Mechanical vibration energy is used to interfere with early events in the biofilm development process viz. the adhesion of planktonic microorganisms to surfaces. By preventing adhesion, the subsequent firm attachment to substrates, gene expression reprogramming, and synthesis of the corresponding protein products that transform the lifestyle of microorganisms from the planktonic to sessile form is aborted.

Ultrasound can be used to inactivate bacteria and deagglomerate bacterial clusters or flocks through a number of physical, mechanical, and chemical effects arising from acoustic cavitation. On collapse, cavitation bubbles produce enough energy to mechanically weaken or disrupt bacteria via a number of processes. The mechanism of microbial killing is mainly due to thinning of cell membranes, localized heating, and production of free radicals. During sonication, longitudinal waves are created when a sonic wave meets a liquid medium, thereby creating regions of alternating compression and expansion. The effect of bacterial inactivation depends on action time, intensity, frequency sensitivity of cells, kind of sonication environment, and presence of additional substances (Piyasena et al., 2003). The mechanism by which lowenergy surface vibration wave interferes with bacterial biofilm formation is based on the hypothesis that low-energy surface vibration wave targets the adhesion of planktonic bacteria to surfaces which is indeed the first step in the biofilm formation process. The increased efficacy of lowenergy surface waves on the anti-adhesive and therefore antimicrobial property of the various MPS is quite evident from the results obtained with and without treatment with these waves (Table 1 and Fig. 1, 2, 3 \& 4). On the whole, utilization of mechanical vibration energy interferes with the early events of the biofilm development process and further prevents subsequent firm attachment of the microorganisms on the surface of contact lens.

This study would therefore help in increasing the level of awareness to choose the correct MPS effective in reducing the risk of ocular infections in the Indian scenario. Further innovations like use of low energy surface vibration waves with MPS which aid the anti adhesive and antimicrobial properties of MPS would definitely prove to be of a great help in prevention of biofilm formation by these ocular isolates and thus help in alleviating the various ocular manifestations amongst contact lens wearers.

\section{References}

Duckhouse, H., Mason, T.J., Phull, S.S., Lorimer, J.P. 2004. The effect of sonication on microbial disinfection using hypochlorite. Ultrason. Sonochem., 11173-176.

Mark Eltis. 2011. Contact-lens-related microbial keratitis: case report and review. J. Optometry, 4(4): 122-127.

Monika Kordowska-Wiater, Dariusz, M., Stasiak. 2011. Effect of ultrasound on survival of Gram-negative bacteria on chicken skin surface. Bull. Vet. Inst. Pulawy., 55: 207-210.

Piyasena, P., Mohareb, E., McKellar, R.C. 2003. Inactivation of microbes using ultrasound: a review. Int. J. Food Microbiol., 87: 207-216.

Tor Monsen, Elisabeth Lövgren, Micael Widerström, Lars Wallinder. 2009. In Vitro Effect of Ultrasound on Bacteria 
and Suggested Protocol for Sonication and Diagnosis of Prosthetic Infections. J. Clin. Microbiol., 47(8): 2496-2501.

Zadik Hazan, Jona Zumeris, Harold Jacob, Hanan Raskin, Gera Kratysh, Moshe Vishnia, Naama Dror, Tilda Barliya,
Mathilda Mandel, Gad Lavie. 2006. Effective Prevention of Microbial Biofilm Formation on Medical Devices by Low-Energy Surface Acoustic waves. Antimicrob. Agents Chemother., 50(12): 4144.

\section{How to cite this article:}

Shilpa Sabnis and Tejas Nair. 2016. Effect of Low Energy Surface Vibration on the Antiadhesive Properties of Multipurpose Solutions. Int.J.Curr.Microbiol.App.Sci. 5(4): 9-14. doi: http://dx.doi.org/10.20546/ijcmas.2016.504.002 